\title{
Composición y diversidad de la vegetación en cuatro sitios del noreste de México
}

\section{Composition and diversity of the vegetation in four sites of Mexico's Northeast}

\author{
Roque G. Ramírez-Lozano', Tilo G. Domínguez-Gómez², \\ Humberto González-Rodríguez ${ }^{2^{*}}$, Israel Cantú-Silva ${ }^{2}$, Marco V. \\ Gómez-Meza ${ }^{3}$, Jorge I. Sarquís-Ramírez ${ }^{4}$ y Enrique Jurado ${ }^{1}$
}

\begin{abstract}
RESUMEN
En el verano de 2007 se determinó la composición y diversidad de la vegetación arbustiva y arbórea en cuatro sitios del estado de Nuevo León. El sitio 1 (S1, Bosque Escuela) a una altitud de $1600 \mathrm{~m}$ se ubicó en el municipio de Iturbide, Nuevo León, México. Los sitios S2 (Crucitas, 550 m), S3 (Campus, 370 m) y S4 (Cascajoso, $300 \mathrm{~m}$ ) se ubicaron en el municipio de Linares, Nuevo León, México. En cada sitio se establecieron aleatoriamente 10 cuadrantes de $10 \mathrm{~m} \times 10 \mathrm{~m}$, en los cuales se estimaron los valores relativos de abundancia, dominancia, frecuencia y valor de importancia (VI) de los árboles y arbustos presentes. Además, se determinaron parámetros dasométricos como altura y diámetro de copa. La diversidad de especies para cada sitio se estimó con el índice de Shannon Wiener. La similitud entre sitios se determinó con el índice de Jaccard. Se registraron un total de 13710 individuos pertenecientes a 28 familias, predominando los géneros y especies de la familia Leguminosae (10) seguidos por Fagaceae (4), Rutaceae (4), Euphorbiaceae (3), Oleaceae (3), Cupressaceae (3), Rhamnaceae (2) y Verbenaceae (2). Dieciocho familias sólo presentaron una especie. El sitio con el mayor y menor índice de Shannon fueron el Cascajoso $(2,08)$ y Bosque Escuela $(1,64)$, respectivamente. El índice de Jaccard sólo mostró igualdad de especies entre S2, S3 y S4. Las especies de plantas más frecuentes fueron: en S1, Quercus canbyi, Pinus pseudostrobus y Rhus pachyrrachys (con 11,9\%); en S2 Havardia pallens (9,9\%); en S3 Havardia pallens y Forestiera angustifolia (con 9,8 \%) y en S4 Acacia rigidula, Cordia boissieri y Karwinskia humboldtiana (con 9,8\%). Los sitios S2 y S3 presentaron la mayor riqueza específica de especies, posiblemente debido a tener mayor precipitación pluvial.
\end{abstract}

PALABRAS CLAVE:

Iturbide, Linares, Nuevo León, parámetros dasométricos, valor de importancia.

\begin{abstract}
During the summer of 2007, a study of the status of trees and woody vegetation was performed at four sites, in the state of Nuevo Leon, Mexico. Site one (S1, Bosque Escuela) was located at $1600 \mathrm{~m}$ elevation in Iturbide county. Sites S2 (Crucitas, $550 \mathrm{~m}$ ), S3 (Campus, $350 \mathrm{~m}$ ) and S4 (Cascajoso, 300 $\mathrm{m}$ ) were located in Linares county. In each site, 10 random plots $(10 \mathrm{~m} \times 10 \mathrm{~m})$ were laid out, and relative abundance, relative dominance and relative frequency were estimated. In addition, dasometric parameters such as height and crown diameter were determined. Plant diversity was estimated by the Shannon-Wiener index, and similarity between sites was calculated using the Jaccard index. A total of 13710
\end{abstract} León, México. 
individual plants belonging to 28 families were registered. Leguminosae had the highest number of species (10) followed by Fagaceae (4), Rutaceae (4), Euphorbiaceae (3), Oleaceae (3), Cupressaceae (3), Rhamnaceae (2) and Verbenaceae (2). Eighteen families included only one species. The site with higher and lower Shannon index was Cascajoso $(2,08)$ and Bosque Escuela $(1,64)$, respectively. The Jaccard index showed similarity among species only among S2, S3 and $\mathrm{S} 4$ sites. The plant species more frequent were: in S1 Quercus canbyi, Pinus pseudostrobus and Rhus pachyrranchys (11,9\%); in S2 Havardia pallens $(9,9 \%)$; in S3 Havardia pallens and Forestiera angustifolia $(9,8 \%)$ and in S4 Acacia rigidula, Cordia boissieri and Karwinskia humboldtiana $(9,8 \%)$. S2 and S3 were the sites with the greatest species diversity, perhaps as a result of higher rainfall.

KEY WORDS:

Iturbide, Linares, Nuevo Leon, dasometric parameters, importance value.

\section{INTRODUCCIÓN}

En Nuevo León hay diferentes tipos de vegetación; destacan los bosques de pino, encino y pino-encino (Vargas, 1999; Silva y González, 2001) y los matorrales subtropicales de las planicies semiáridas. Este último tipo de vegetación, denominado Matorral Espinoso Tamaulipeco, está constituido de especies deciduas y siempre verdes o perennes (Reid et al., 1990) y está caracterizado por una amplia variedad de patrones de crecimiento, así como diversidad en la longevidad foliar que se debe al papel de varios factores del medio que influyen en la estructura de las comunidades vegetales: 1) dentro de sitios, entre sitios de una región o entre regiones (Menge y Olson, 1990); 2) el paisaje que juega un papel importante en la organización de las comunidades (Valverde et al.,1996); 3) el clima que influye en la organización de las comunidades (González-Medrano, 1996), 4) los componentes bióticos relacionados con la dispersión de semillas (Jerry et al., 2002) y 5) los factores edáfi- cos (Abd El-Ghani, 2000; Yoder y Nowak, 2000).

En las regiones semiáridas, como el noreste de México, la composición florística y la estructura de la vegetación están fuertemente determinadas por el contenido de humedad del suelo disponible para desarrollo de las plantas (Reid et al., 1990), a tal grado que se reconoce como el principal factor limitante en estas regiones. El agua disponible para las plantas no solamente está relacionada con la precipitación anual, sino también con las propiedades físicas del suelo y la topografía, que modifican la capacidad de retención del agua, percolación y las tasas de evaporación de los sustratos (GonzálezRodríguez et al., 2009). Asimismo, los disturbios naturales y antropogénicos frecuentemente alteran la composición florística y la estructura de la vegetación (Jiménez, 2009). Por lo tanto, es importante complementar los estudios de vegetación con una clasificación de las especies dentro de un espectro de formas de vida y biológicas que provea componentes estructurales de los conjuntos de vegetación (Batalha y Martins, 2004).

\section{OBJETIVOS}

Este estudio tuvo como objetivos describir y comparar la composición y diversidad de plantas arbóreas y arbustivas en cuatro áreas del estado de Nuevo León con diferente nivel de altitud.

\section{METODOLOGÍA}

\section{Localización y descripción de los sitios de estudio}

El estudio se llevó a cabo durante el verano de 2007 en cuatro sitios ubicados en el estado de Nuevo León, México (Fig. 1). La distancia entre sitios fue la siguiente: entre 

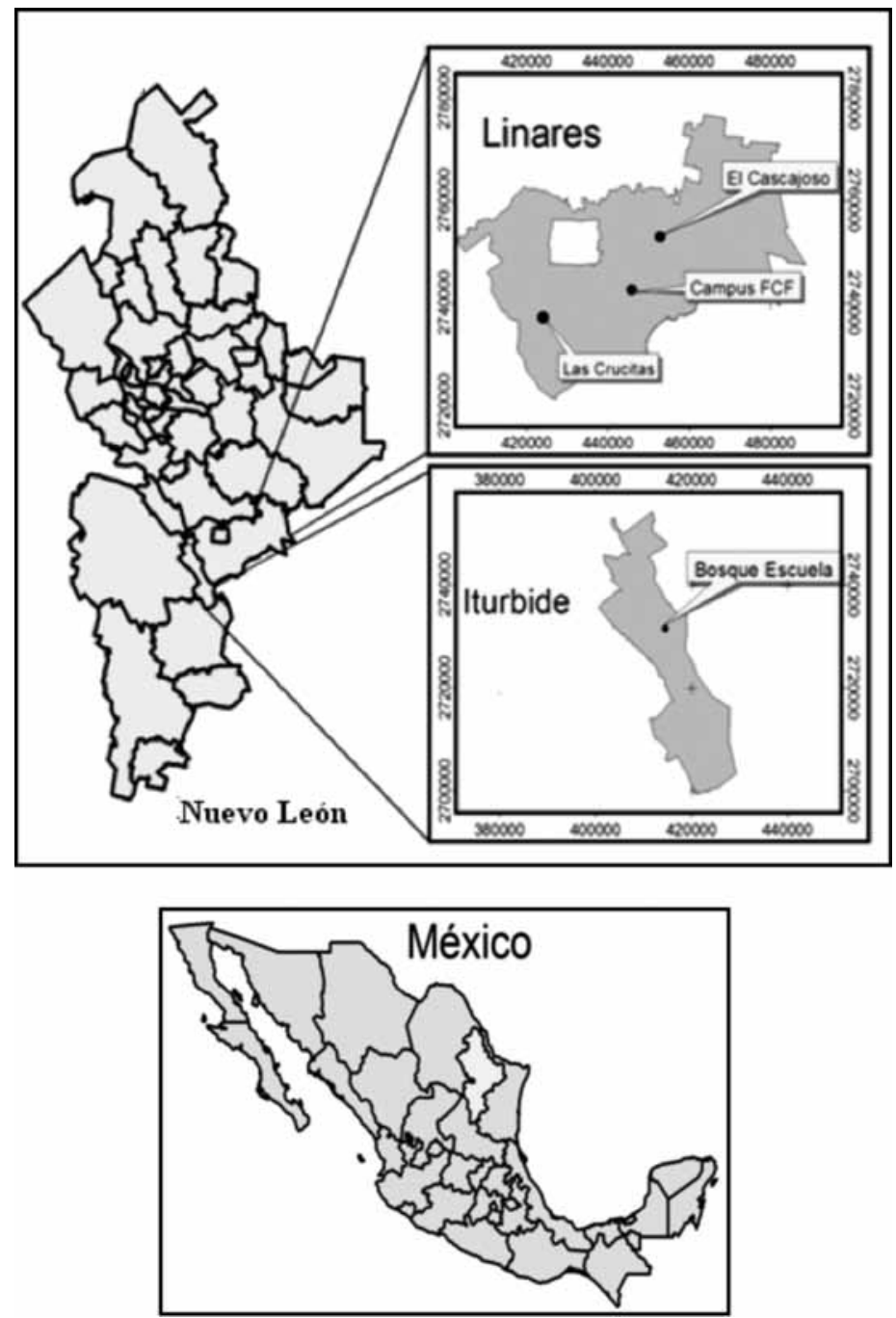

Figura 1. Localización de los sitios de estudio.

$\mathrm{S} 1$ y S2 $35 \mathrm{~km}$, de S2 a S3 $28 \mathrm{~km}$ y entre S3 y S4 $29 \mathrm{~km}$. En la tabla 1 se muestra la descripción de los sitios de estudio.

\section{Evaluación de la vegetación}

En cada sitio se establecieron al azar 10 unidades de muestreo de $10 \mathrm{~m} \times 10 \mathrm{~m}$ en un área de aproximadamente $2500 \mathrm{~m}^{2}$. En cada unidad se evaluaron todas las especies arbóreas y arbustivas presentes. A cada ejemplar se le determinaron los parámetros dasométricos de altura total $(\mathrm{m}) \mathrm{y}$ cobertura $\left(\mathrm{m}^{2}\right)$ de copas; este último se estimó tomando la longitud del largo (nortesur) por ancho (oriente-poniente) de cada copa. 
Tabla 1. Descripción de los cuatro sitios de estudio.

\begin{tabular}{|c|c|c|c|c|}
\hline \multicolumn{5}{|c|}{ Sitio de estudio } \\
\hline & S1 & S2 & S3 & S4 \\
\hline Caracteristicas & Bosque Escuela & Crucitas & Campus & Cascajoso \\
\hline \multirow[t]{2}{*}{ Coordenadas } & $24^{\circ} 42^{\prime} 28^{\prime \prime} \mathrm{N}$ & $24^{\circ} 46^{\prime} 35^{\prime \prime} \mathrm{N}$ & $24^{\circ} 46^{\prime} 43^{\prime \prime} \mathrm{N}$ & $24^{\circ} 54^{\prime} 17^{\prime \prime} \mathrm{N}$ \\
\hline & $99^{\circ} 5^{\prime} 43^{\prime \prime} \mathrm{O}$ & $99^{\circ} 4^{\prime} 44^{\prime \prime} \mathrm{O}$ & $99^{\circ} 31^{\prime} 39^{\prime \prime} \mathrm{O}$ & $99^{\circ} 25^{\prime} 43^{\prime \prime} \mathrm{O}$ \\
\hline Altitud (msnm) & 1600 & 550 & 370 & 300 \\
\hline \multirow[t]{2}{*}{ Vegetación } & bosques de & bosque pino-encino & MET & MET \\
\hline & pino-encino & y matorral alto subinerme & & \\
\hline $\begin{array}{l}\text { Temperatura } \\
\text { media anual }\end{array}$ & $14^{\circ} \mathrm{C}$ & $21^{\circ} \mathrm{C}$ & $14^{\circ} \mathrm{C}$ & $12^{\circ} \mathrm{C}$ \\
\hline $\begin{array}{l}\text { Precipitación } \\
\text { media anual }\end{array}$ & $635 \mathrm{~mm}$ & $755 \mathrm{~mm}$ & $805 \mathrm{~mm}$ & $672 \mathrm{~mm}$ \\
\hline Suelo & $\begin{array}{c}\text { Castañozem } \\
\text { y Leptosol }\end{array}$ & $\begin{array}{l}\text { Castañozem } \\
\text { y Chernozem }\end{array}$ & Vertisol & $\begin{array}{l}\text { Vertisol } \\
\text { Calcárico }\end{array}$ \\
\hline
\end{tabular}

FUENTE: (SPP-INEGI, 1986; Bravo Garza, 1999; INEGI, 2001).

\section{Análisis de la información}

Se determinaron los indicadores ecológicos como: abundancia (A), dominancia $(D)$, frecuencia $(F)$ y valor de importancia (VI). La diversidad de especies se determinó mediante el índice Shannon Wiener, uno de los más frecuentes para determinar la diversidad de plantas de un hábitat (Matteucci et al., 1999). Para determinar la similitud entre sitios de muestreo se utilizó el índice de Jaccard, cuyos datos cualitativos están basados en la presencia o ausencia de las especies en los sitios (Magurran, 1988). En este estudio, para estimar la dominancia de las especies se utilizaron los datos de área de copa en lugar del área basal.

Los datos obtenidos con el índice de Shannon fueron analizados estadísticamente. Los resultados no presentaron homogeneidad de varianzas (prueba de Levene a $\mathrm{P}=0,05)$, pero sí mostraron una distribución normal de acuerdo con las pruebas estadísticas de KolmogorovSmirnov (con la corrección de Lilliefors) y Shapiro-Wilk (Brown y Forsythe, 1974); por tanto, los datos fueron analizados mediante la prueba no paramétrica de Kruskal-Wallis con la corrección de Bonferroni (Steel y Torrie, 1980; Ott, 1993) para detectar diferencias estadísticas en el índice de Shannon entre los sitios.

Los datos de densidad (n/ha) no mostraron distribución normal según las pruebas de Kolmogorov-Smirnov (con la corrección de Lilliefors) y Shapiro-Wilk, tampoco mostraron homogeneidad de varianzas (prueba de Levene; $P=0,05$ ). Por lo anterior, los datos fueron analizados mediante la prueba no paramétrica de Kruskal-Wallis con la corrección de 
Bonferroni. Los datos de cobertura no mostraron distribución normal (prueba de Shapiro-Wilk), por lo que se analizaron a través de la prueba no paramétrica de Kruskal-Wallis con la corrección de Bonferroni.

Todos los procedimientos estadísticos fueron realizados mediante el uso del paquete estadístico SPSS versión 13.0.

\section{RESULTADOS Y DISCUSIÓN}

\section{Composición florística}

Se registraron un total de 13710 individuos pertenecientes a 28 familias, predominando las plantas de la familia Leguminosae (10) seguidas por Fagaceae (4), Rutaceae (4), Euphorbiaceae (3), Oleaceae (3), Cupressaceae (3), Rhamnaceae

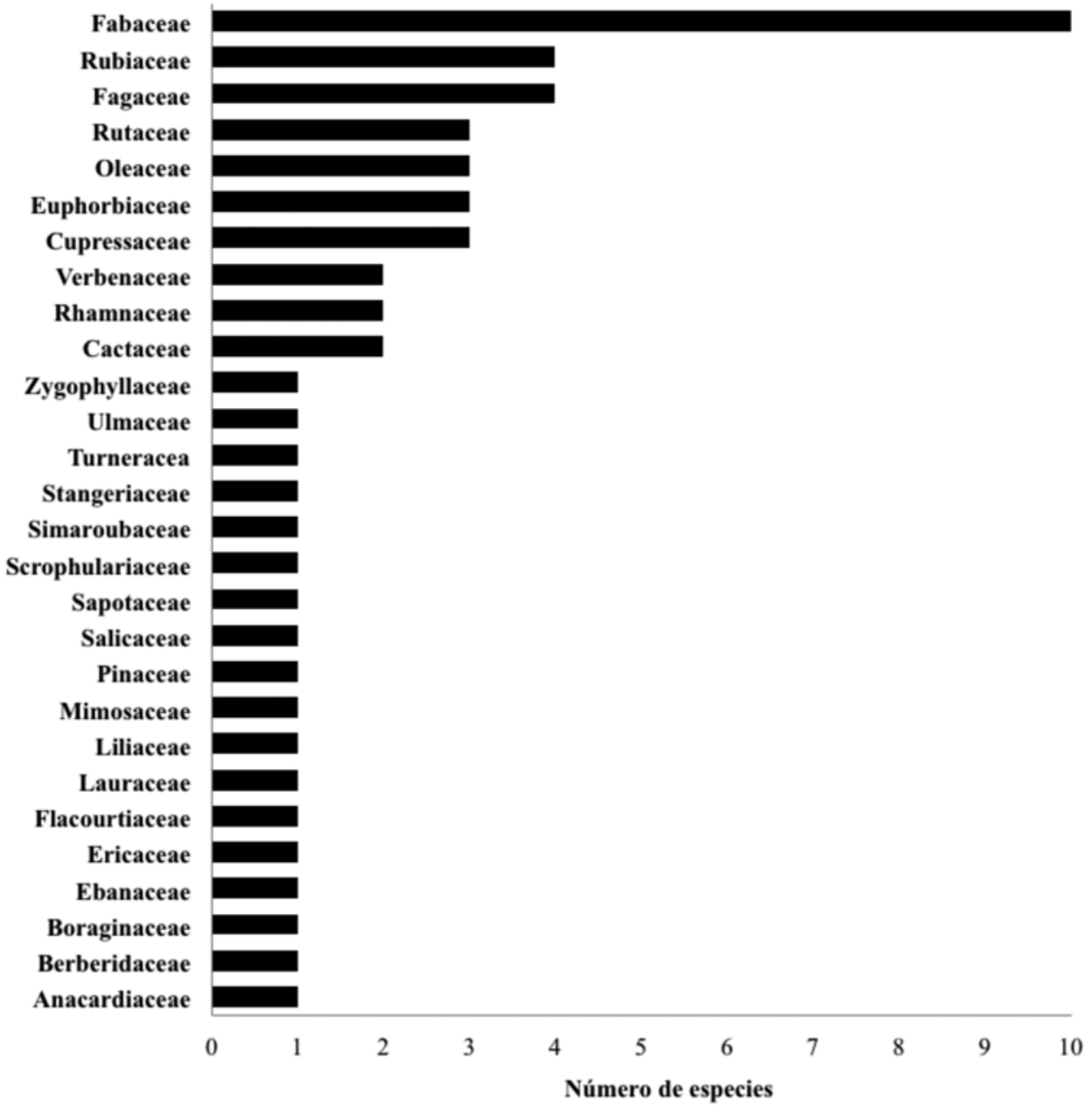

Figura 2. Distribución del número de especies por familia, en los cuatro sitios de estudio. 
(2) y Verbenaceae (2). En las restantes 18 familias sólo se presentó una especie por familia (Fig. 2). La alta presencia de individuos de la familia Leguminosae como Acacia farnesiana y $A$. rigidula, especies presentes en tres sitios (S2, S3 y S4), se debe probablemente a que en el estado de Nuevo León, algunos taxa son abundantes sobre grandes extensiones, como Acacia rigidula, A. constricta, A. berlandieri, A. farnesiana y Prosopis glandulosa, asociados a diversas comunidades de matorral xerófilo. Asimismo, factores tales como la exigua disponibilidad de nutrientes en el suelo, intolerancia a la sombra y mecanismos relacionados con la reproducción pueden incrementar el número de individuos de la familia Leguminosae (García y Jurado, 2008; Jiménez-Pérez et al., 2009).

De acuerdo con el análisis estadístico, para los datos de densidad no se detectaron diferencias significativas $\left(\chi^{2}=0,495 ; \mathrm{P}=0,922\right)$ entre los sitios. La densidad para los sitios $1,2,3$ y 4 fue 3,$850 ; 3,120 ; 3,360$ y 3,380 (individuos/ ha), respectivamente.

La prueba de Kruskal-Wallis mostró que los datos obtenidos de diversidad fueron significativamente diferentes entre sí, con valores de 1,$64 ; 1,92 ; 2,02$ y 2,08 para S1, S2, S3 y S4, respectivamente. Resultados similares $(2,6 ; 2,8$ y 2,4 para $S 1, S 2$ y S3, respectivamente) fueron encontrados por González-Rodríguez et al. (2010), quienes cuantificaron la diversidad de especies en tres sitos localizados en el matorral espinoso tamaulipeco (MET) del estado de Nuevo León. Asimismo, Espinoza y Návar (2005), en cinco sitios con diferente nivel de productividad en el MET, encontraron valores de 2,8; 2,5; 2,7; 3,2 y 2,9 y argumentan que la semejanza en los índices de Shannon pudiera deberse a que los cambios en la riqueza de especies se equilibran con los cambios en la abundancia. Es decir, mientras que el gradiente de riqueza de especies se mueve en una dirección, la abundancia se mueve en otra dirección.

La diversidad en los sitios de muestreo, en general es caracterizada como intermedia en comparación con determinaciones similares obtenidas en análisis tipo para la región (González-Rodríguez et al., 2010). Lo anterior pudiera significar que el ecosistema de esa región tiene relevancia ecológica media, con fuertes variaciones en la tendencia de la diversidad de las especies, debida en parte a las condiciones climáticas, físicas y ecológicas del hábitat de la región y posibles cambios en uso del suelo hacia la ganadería extensiva (Alanís et al., 2008).

El índice de Jaccard (Tabla 2) muestra que las especies del $\mathrm{S} 1$ son muy diferentes a las de $S 2(0,02), S 3(0,00)$ y $S 4$ $(0,06)$. Sin embargo, al comparar S2 con S3 $(0,47)$ y con $S 4(0,21)$ y S3 con $S 4$ $(0,33)$ tienen mayor similitud de especies. Lo que significa que el S2, S3 y S4 son similares en su composición de especies y

Tabla 2. Índices de similitud florística de Jaccard entre sitios.

\begin{tabular}{llccc}
\hline & \multicolumn{2}{c}{ Sitio de estudio } \\
& Bosque Escuela (S1) & Crucitas (S2) & Campus (S3) & Cascajoso (S4) \\
\hline S2 & 0,02 & & \\
S3 & 0,00 & 0,47 & \\
S4 & 0,06 & 0,21 & 0,33 \\
\end{tabular}




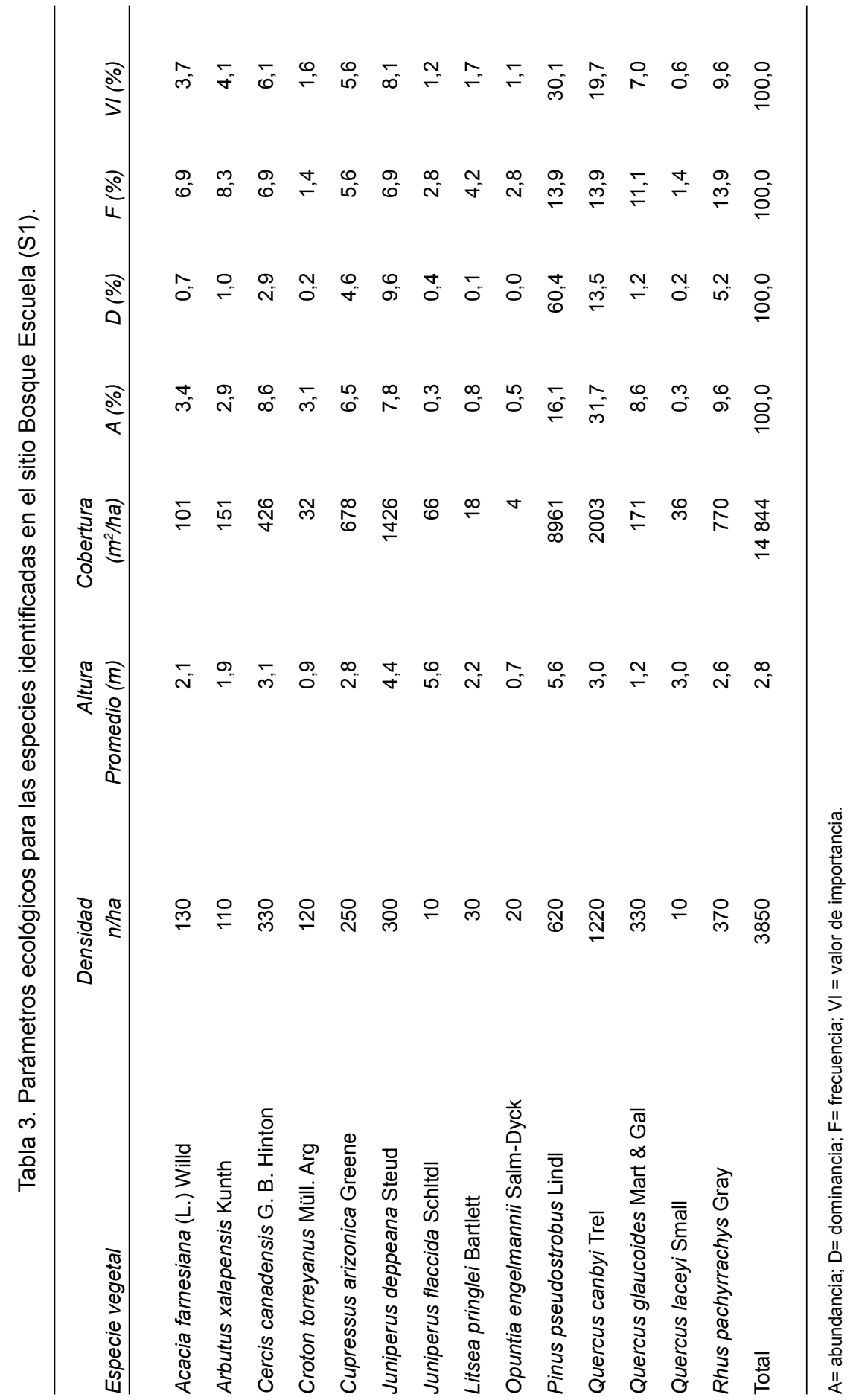




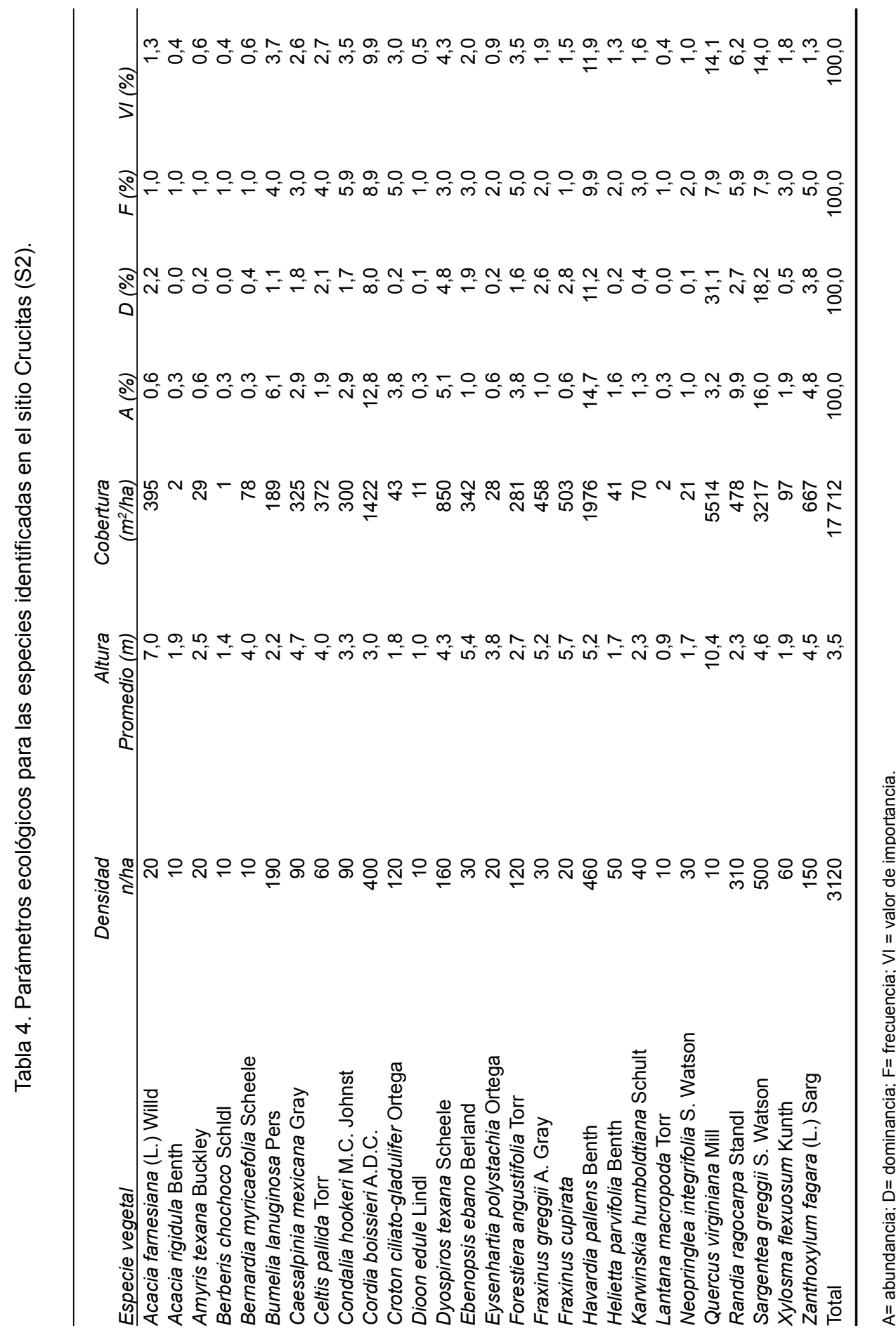




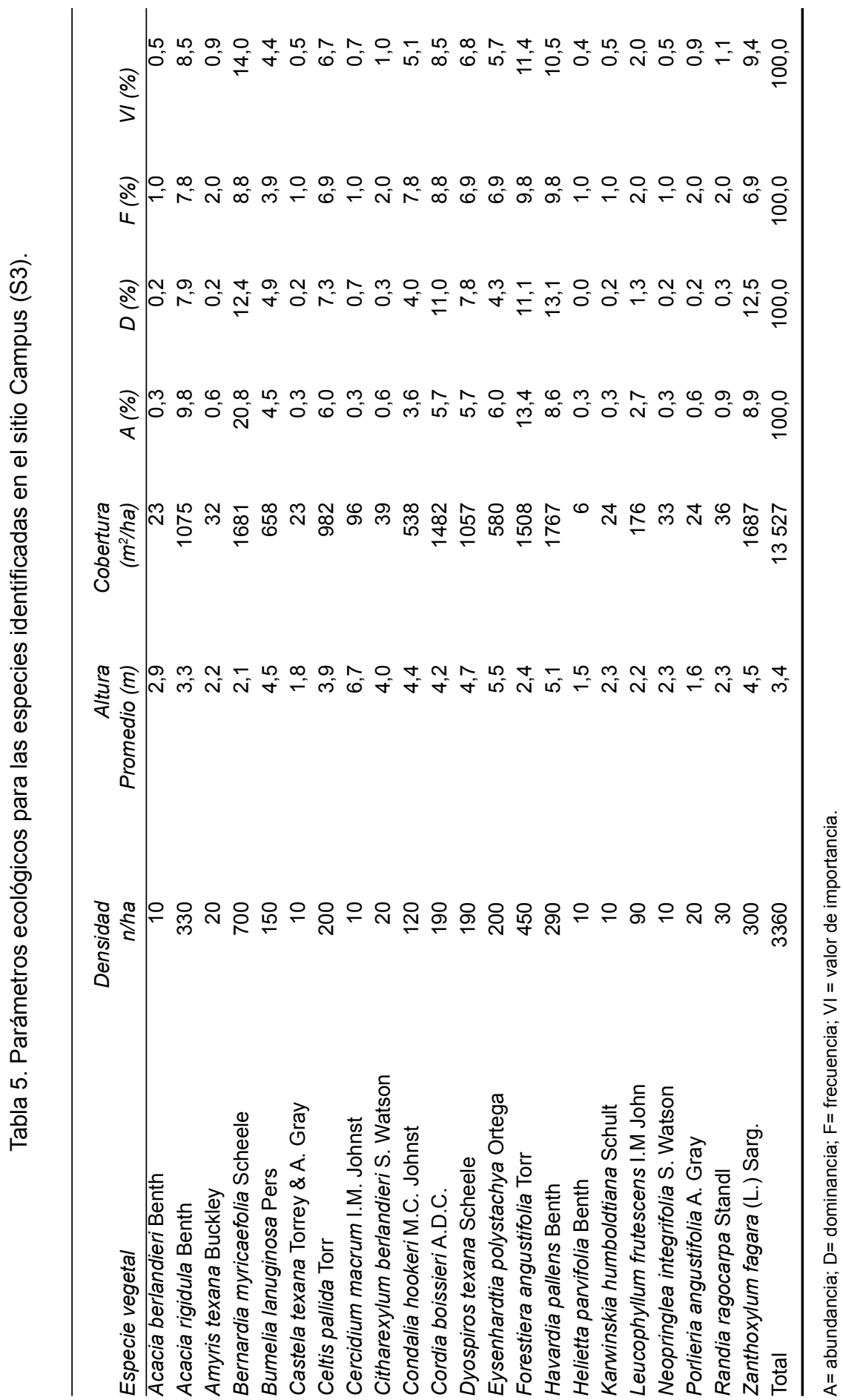




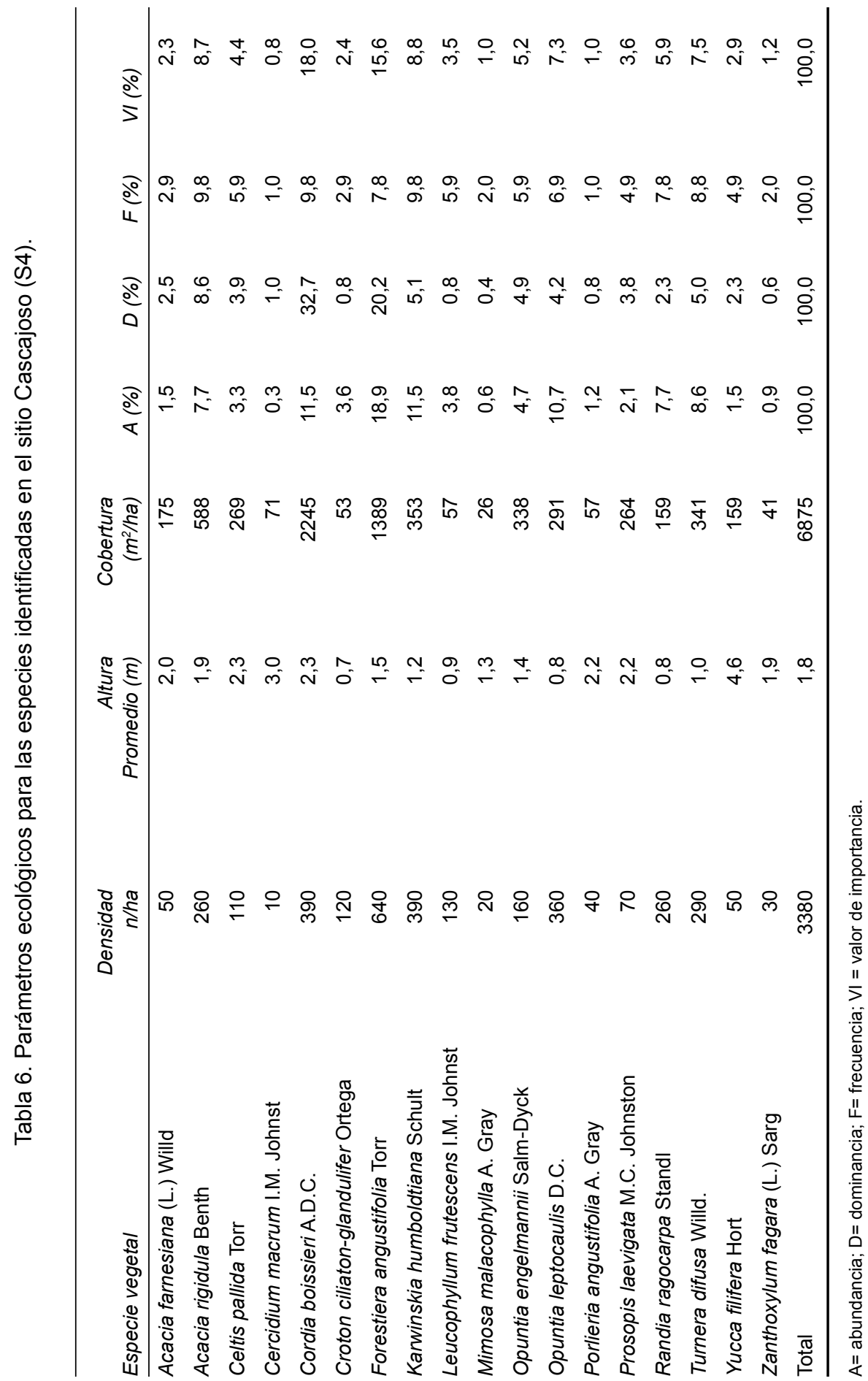


destacan por su mayor número de individuos, lo cual los distingue del S1, quizá por la precipitación históricamente registrada. El S1 sobresale por su menor número de individuos y con más especies arbóreas que arbustivas, tal vez por la mayor altitud a la que se encuentra y las características de este ecosistema que lo hace diferente al de los demás sitos evaluados.

\section{Indicadores ecológicos}

Con respecto a los datos de cobertura, la prueba de Kruskal-Wallis detectó diferencias significativas $\left(\chi^{2}=20,18 ; \quad P=0,001\right)$ entre los sitios de estudio. Las mayores coberturas correspondieron a S1, S2 y S3 (Tablas 3-5) con valores de $14844 \mathrm{~m}^{2} / \mathrm{ha}$, $17712 \mathrm{~m}^{2} /$ ha y $15527 \mathrm{~m}^{2} / \mathrm{ha}$, respectivamente; mientras que el sitio de menor cobertura correspondió a S4 (Tabla 6) con $6875 \mathrm{~m}^{2} / \mathrm{ha}$. Los individuos del S1 tuvieron una altura promedio de 2,8 m (Tabla 3). Las plantas con mayor altura fueron Pinus pseudostrobus y Juniperus flaccida y la menor fue Opuntia engelmannii. La especie Pinus pseudostrobus tuvo la mayor cobertura (8961 $\left.\mathrm{m}^{2} / \mathrm{ha}\right)$ y Opuntia engelmannii $\left(4 \mathrm{~m}^{2} / \mathrm{ha}\right)$ la menor. Quercus canbyi fue la más abundante y Opuntia engelmannii la menos abundante. La especie dominante fue Pinus pseudostrobus y Croton torreyanus fue la especie con menor dominancia. Quercus canbyi también fue la especie más frecuente junto con Pinus pseudostrobus. Esta última tuvo el mayor valor de importancia y Quercus laceyi presentó el menor (Tabla 3). Las especies del S2 tienden a presentar mayor altura (3,5 $\mathrm{m})$ comparadas con las de otros sitios. La especie con mayor altura $(10,4 \mathrm{~m})$ y cobertura $\left(5515 \mathrm{~m}^{2} / \mathrm{ha}\right)$ fue Quercus virginiana y la menor fue Lantana macropoda (0,9 $\mathrm{m}$ y $2,0 \mathrm{~m}^{2} / \mathrm{ha}$, respectivamente). La especie más abundante fue Sargentea greggii mientras que Acacia rigidula, Barberis chochoco, Bernardia myricaefolia y Dioon edule fueron las menos abundantes. Quercus virginiana fue le especie dominante y Acacia rigidula y Lantana macropoda las especies con menor dominancia. La planta más frecuente fue Cordia boissieri y las menos frecuentes fueron Acacia farnesiana, A. rigidula, Amyris texana, Berberis chochoco y Bernardia myricaefolia. Sargentea greggii tuvo el mayor valor de importancia y Acacia rigidula, Berberis chochoco y Lantana macropoda tuvieron el menor valor de importancia (Tabla 4). Las plantas del S3 mostraron en promedio la segunda mayor altura $(3,4 \mathrm{~m})$ con cobertura de $13527 \mathrm{~m}^{2} / \mathrm{ha}$. La especie con mayor altura fue Cercidium macrum $(6,7 \mathrm{~m})$ y la menor fue Helietta parvifolia (1,5 m). La especie con mayor cobertura fue Havardia pallens $\left(1767 \mathrm{~m}^{2} / \mathrm{ha}\right)$ y la menor fue Helietta parvifolia $\left(6 \mathrm{~m}^{2} / \mathrm{ha}\right)$. Bernardia myricaefolia fue la especie más abundante mientras que Amyris texana y Porlieria angustifolia fueron las menos abundantes. Havardia pallens resultó la de mayor densidad y Castela texana la de menor. Forestiera angustifolia y Havardia pallens fueron las más frecuentes y Cercidium macrum la menos frecuente. Bernardia myricaefolia tuvo el mayor valor de importancia y Helietta parvifolia el menor (Tabla 4). Las especies del S4 presentaron, en promedio, la menor altura $(1,8 \mathrm{~m})$ y la menor cobertura $\left(6875 \mathrm{~m}^{2} / \mathrm{ha}\right)$. La especie con mayor altura fue Yucca filifera $(4,6 \mathrm{~m})$ y Croton ciliaton-glandulifer $(0,7 \mathrm{~m})$ la menor. Cordia boissieri resultó con la mayor cobertura $\left(2245 \mathrm{~m}^{2} / \mathrm{ha}\right)$ y Mimosa malacophylla fue menor $\left(26 \mathrm{~m}^{2} / \mathrm{ha}\right)$. Forestiera angustifolia fue la especie más abundante y Mimosa malacophylla fue menor. La de mayor densidad fue Cordia boissieri y la menor fue Croton ciliaton-glandulifer. Cordia boissieri también mostró la mayor frecuencia mientras que Cercidium macrum y Porlieria angustifolia mostraron la menor. La especie con mayor valor de importancia fue Cordia boissieri y la de menor fue Cercidium macrum (Tabla 5). 


\section{CONCLUSIONES}

Las especies de plantas que presentaron el mayor número de individuos fueron: en S1 Quercus canbyi y Pinus pseudostrobus; en S2 Havardia pallens y Cordia boissieri; en S3 Bernardia myricaefolia y Forestiera angustifolia; en S4 Forestiera angustifolia, Cordia boissieri y Karwinskia humboldtiana. Las especies de plantas con mayor cobertura fueron: en S1 Pinus pseudostrobus y Quercus canbyi; en S2 Quercus virginiana y Sargentea greggii; en S3 Havardia pallens y Zanthoxylum fagara; en S4 Cordia boissieri y Forestiera angustifolia. Las especies de plantas que presentaron los mayores valores de importancia fueron: en S1 Pinus pseudostrobus y Quercus canbyi; en S2, Quercus virginiana, Sargentea greggii y Havardia pallens; en S3, Bernardia myricaefolia y Forestiera angustifolia y en S4, Cordia boissieri y Forestiera angustifolia. Los cuatro sitios mostraron la misma fitodiversidad; sin embargo, S1, localizado a mayor altitud presentó una composición vegetal diferente a los otros sitios. Por tanto, se concluye que factores climatológicos y topográficos tuvieron influencia sobre la composición vegetal, ya que las especies presentaron diferencias en su valor de importancia en los sitios evaluados. Debido a las condiciones medianas de la diversidad de las especies en todos los sitios, sería importante realizar estudios que promuevan la expansión de la vegetación que ayudarían a la sostenibilidad de los ecosistemas de la región.

\section{REFERENCIAS}

Abd El-Ghani, M.M. 2000. Floristics and environmental relations in two extreme desert zones of western Egypt. Global Ecology and Biogeography 9:499-516.
Alanís, E., J. Jiménez, O.A. Aguirre, E.J. Treviño, E. Jurado y M.A. González. 2008. Efecto del uso del suelo en la fitodiversidad del matorral espinoso tamaulipeco. Revista Ciencia UANL, $\mathrm{XI}: 56-62$.

Batalha, M.A. y F.R. Martins. 2004. Floristic, frequency, and vegetation lifeform spectra of a cerrado site. Brazilian Journal of Biology 64:2-10.

Bravo Garza, M.R. 1999. Distribución de la materia orgánica del suelo en ecosistemas naturales e inducidos en el Estado de Nuevo León. Tesis de Licenciatura. Facultad de Ciencias Forestales, UANL. Linares, NL. 84 p.

Brown, M.B. y A.B. Forsythe. 1974. Robust tests for the equality of variances. Journal of the American Statistical Association 69:364-367.

Espinoza-Bretado, R. y J. Návar. 2005. Producción de biomasa, diversidad y ecología de especies en un gradiente de productividad en el matorral espinoso tamaulipeco del Nordeste de México. Revista Chapingo Serie Ciencias Forestales y del Ambiente 11:25-35.

García, J. y E. Jurado. 2008. Caracterización del matorral con condiciones prístinas en Linares N.L., México. Ra Ximhai 4:1-21.

González-Medrano, F. 1996. Algunos aspectos de la evolución de la vegetación de México. Boletín de la Sociedad Botánica de México 58:129-136.

González-Rodríguez, H., I. Cantú-Silva, M.V. Gómez-Meza y W.R. Jordan. 2000. Seasonal plant water relationships in Acacia berlandieri. Arid Soil Research and Rehabilitation 14:343357. 
González, R.H. y C.I. Silva. 2001. Adaptación a la sequía de plantas arbustivas del matorral espinoso Tamaulipeco. Revista Ciencia UANL IV:454-461.

González-Rodríguez, H., I. Cantú-Silva, M.V. Gómez-Meza y R.G. RamírezLozano. 2004. Plant water relations of thornscrub shrub species, northeastern Mexico. Journal of Arid Environments 58:483-503.

González-Rodríguez, H., I. Cantú-Silva, M.V. Gómez-Meza, R.G. RamírezLozano, M. Pando-Moreno, I.A. Molina-Camarillo y R.K. Maiti. 2009. Water relations in native trees, northeastern Mexico. International Journal of Agriculture, Environment and Biotechnology II:133-141.

González-Rodríguez, H., R.G. RamírezLozano, I. Cantú-Silva, M.V. GómezMeza y J.I. Uvalle-Sauceda. 2010. Composición y estructura de la vegetación en tres sitios del estado de Nuevo León, México. Polibotánica 29:91-106.

INEGI (Instituto Nacional de Estadística y Geografía). 2001. Cartas topográficas y edafología de Linares 1:50,000G14C59, Segunda Edición.

Jerry, F.F., A.S. Thomas, U.P. Robert, B.C. Andrew y A.T. Dale. 2002. Disturbances and structural developments of natural forest ecosystems with silvicultural implications using Douglas fir forest as an example. Forest Ecology and Management 155:399-423.

Jiménez-Pérez, J., E. Alanís-Rodríguez, O. Aguirre-Calderón, M. PandoMoreno y M. González-Tagle. 2009. Análisis sobre el efecto del uso del suelo en la diversidad estructural del matorral espinoso tamaulipeco. Madera y Bosques 15(3):5-20.

Magurran, A.E. 1988. Ecological diversity and its measurement. Princeton University Press. Nueva Jersey. 179 p.

Matteucci, S.D., A. Colma y L. Pla. 1999. Biodiversidad vegetal en el árido falconiano (Venezuela). Interciencia 24:300-307.

Menge, B.A. y A.M. Olson. 1990. Role of scale and environmental factors in regulation of community structure. Trends in Ecology and Evolution 5:52-57.

Ott, L. 1993. An introduction to statistical methods and data analysis. 2a. ed. Duxbury Press. Boston, Massachusetts p:775-801.

Reid, N., J. Marroquín y M.P. Beyer. 1990. Utilization of shrubs and trees for browse, fuelwood and timber in the Tamaulipan thornscrub, northeastern Mexico. Forest Ecology and Management 36:61-79.

Ramírez, R.G., H. González-Rodríguez, R. Ramírez-Orduña, M.A. CerrilloSoto y A. Juárez-Reyes. 2006. Seasonal trends of macro and microminerals in 10 browse species that grow in northeastern Mexico. Animal Feed Science and Technology 128:155-164.

Silva, C.I. y R.H. González. 2001. Interception loss, throughfall and stem flow chemistry in pine and oak forests in northeastern Mexico. Tree Physiology 21:1009-1013.

SPP-INEGI (Secretaría de Programación y Presupuesto - Instituto Nacional de Estadística y Geografía). 1986. Sín- 
tesis geográfica del Estado de Nuevo León. SPP-INEGI. México.

Steel, R.G.H. y J.H. Torrie. 1980. Principles and procedures of statistics. 2a. ed. McGraw-Hill Book Co. Nueva York. p:107-133.

Stienen, H., M.P. Smits, N. Reid, J. Landa y J.H.A. Boerboom. 1989. Ecophysiology of 8 woody multipurpose species from semiarid northeastern Mexico. Annales des Sciences Forestièrs 46:454-458.

Valverde, P.L., J.A. Zavala-Hurtado, C. Montaña y E. Escurra. 1996. Numerical analyses of vegetation based on environmental relationships in the southern Chihuahuan Desert. Southwestern Naturalist 41:424-433.

Vargas, L.B. 1999. Caracterización de la productividad y estructura de Pinus hartwegii Lindl en tres gradientes altitudinales en el cerro Potosí, Galeana, Nuevo León. Tesis de Maestría en Ciencias Forestales. Facultad de Ciencias Forestales. UANL. Linares, Nuevo León. 93 p.

Yoder, C.K. y R.S. Nowak. 2000. Phosphorus acquisition by ssp. from soils interspaces shared with Mojave Desert shrubs. Functional Ecology 14:685-692. quís-Ramírez y E. Jurado. 2013. Composición y diversidad de la vegetación en cuatro sitios del noreste de México. Madera y Bosques 19(2):59-72. 